\title{
Suboptimal Immunization Coverage among Canadian Rheumatology Patients in Routine Clinical Care
}

\author{
Tedi Qendro, María Laura de la Torre (D), Pantelis Panopalis, Elizabeth Hazel, Brian J. Ward (1), \\ Inés Colmegna $\mathbb{B}^{\mathbb{D}}$, and Marie Hudson
}

ABSTRACT. Objective. To assess vaccination coverage and predictors of vaccination among a Canadian population of rheumatology patients in routine clinical care.

Methods. In this cross-sectional study, consecutive adult patients presenting to a tertiary rheumatology clinic at the McGill University Health Center between May and September 2015 were asked to fill a survey on vaccination. Patients self-identified as having rheumatoid arthritis (RA), systemic autoimmune rheumatic diseases (SARD), spondyloarthropathies (SpA), or other diseases (OD). Multivariate logistical regression analyses were performed to evaluate patient and physician factors associated with various vaccinations [for influenza, pneumococcus, and hepatitis B virus (HBV)]. Published Quebec general population influenza and pneumococcal vaccination rates in those aged $\geq 65$ years were used as comparative baseline rates.

Results. Three hundred fifty-two patients were included in the analysis (RA: 136, SARD: 113, SpA: 47, OD: 56). Vaccination rates were reported as follows: (1) influenza: RA 48.5\%, SARD 42.0\%, SpA $31.9 \%$, OD $88.9 \%$, Quebec general population 58.5\%; (2) pneumococcal: RA 42.0\%, SARD 37.8\%, SpA $29.7 \%$, OD 33.3\%, Quebec general population 53.2\%; (3) HBV: RA 33.6\%, SARD 55.6\%, SpA $73.5 \%$, OD 36.8\%; and (4) herpes zoster: RA 5.6\%, SARD 28.6\%, SpA 25.0\%, OD 16.7\%. Physician recommendation was the strongest independent predictor of vaccination across all vaccine types (influenza: OR 8.56, 95\% CI 2.80-26.2, p < 0.001; pneumococcal: OR 314, 95\% CI 73.0-1353, $\mathrm{p}<0.001$; HBV: OR 12.8, 95\% CI 5.27-31.1, p < 0.001). Disease group, disease duration, comorbidities, treatment type, and being followed by a primary care physician were not significantly associated with vaccination.

Conclusion. There is suboptimal immunization coverage among ambulatory rheumatology patients. An important role for patient and physician education is highlighted in our study, especially because physician recommendation of vaccination was strongly predictive of vaccine uptake. (First Release December 1 2019; J Rheumatol 2020;47:770-8; doi:10.3899/jrheum.181376)

Key Indexing Terms:

VACCINATION COVERAGE

PREVENTIVE HEALTH SERVICES

RHEUMATIC DISEASES
CANADA

From the Department of Medicine, McGill University, Montreal; Division of Rheumatology, McGill University Health Center, Montreal; Infectious Diseases and Immunity in Global Health Program, Research Institute of the McGill University Health Center, Montreal; Division of Rheumatology and Lady Davis Institute for Medical Research, Jewish General Hospital, Montreal, Canada; Internal Medicine, Rheumatology and Immunology, Centro de Educacion Medica e Investigaciones Clinicas Norberto Quirno, Buenos Aires, Argentina.

This research was in part supported by the Canadian Rheumatology Association and a 2016 Canadian Institute for Outcomes in Rheumatology Care grant on "Strategies to enhance influenza/pneumococcal vaccination coverage among rheumatoid arthritis patients."

T. Qendro, MSc, Department of Medicine, McGill University; M.L. de la Torre, MD, Internal medicine, Rheumatology and Immunology, Centro de Educacion Medica e Investigaciones Clinicas Norberto Quirno; P. Panopalis, MD, Division of Rheumatology, McGill University Health Center; E. Hazel, MD, Division of Rheumatology, McGill University Health Center; B.J. Ward, MD, DTM\&H, Infectious Diseases and Immunity in Global Health Program, Research Institute of the McGill University Health Center; I. Colmegna, MD, Division of Rheumatology, McGill University Health Center; M. Hudson, MD, MPH, FRCPC, Division of Rheumatology and Lady Davis Institute for Medical Research, Jewish General Hospital.

\author{
Address correspondence to Dr. M. Hudson, Jewish General Hospital and \\ Lady David Research Institute, 3755 Côte Ste-Catherine Road, \\ Room A725, Montreal, Quebec H3T 1E2, Canada. \\ E-mail:marie.hudson@mcgill.ca \\ Accepted for publication June 25, 2019.
}

Compared to the general population, patients with rheumatic diseases (RD) have an increased risk of infection and infection-related morbidity and mortality ${ }^{1}$. Contributing to the increased risk from infectious diseases are immune dysfunction due to disease-specific processes, the use of immunosuppressive or biologic agents targeting key components of immunity, and disease-related comorbidities ${ }^{2}$. Consequently, infection prevention is an important goal in the treatment of patients with RD. Vaccines are available for several infectious agents, and they provide an effective opportunity for prophylaxis.

Respiratory tract infections caused by influenza virus and Streptococcus pneumoniae are among the most important

Personal non-commercial use only. The Journal of Rheumatology Copyright ( $) 2020$. All rights reserved. 
infectious risks in patients with $\mathrm{RD}^{3,4}$. A retrospective cohort study of 46,030 rheumatoid arthritis (RA) patients and a matching number of controls from the United States demonstrated a higher incidence of influenza and its complications in patients with $\mathrm{RA}^{5}$. A similar study in the United Kingdom showed an increased likelihood of hospital admission for pneumococcal disease in patients with RA, systemic sclerosis (SSc), Sjögren syndrome, and systemic lupus erythematosus (SLE) compared to the general population ${ }^{6}$. Importantly, influenza and pneumococcal vaccines have not been shown to trigger autoimmune disease activity in patients with $\mathrm{RD}^{1,2,3}$.

Patients with RD also have an increased risk of developing herpes zoster (HZ) that is further elevated with exposure to corticosteroids, nonbiological disease-modifying antirheumatic drugs (DMARD), tumor necrosis factor- $\alpha$ (TNF- $\alpha$ ) blockers, and Janus kinase inhibitors ${ }^{1,7,8,9}$. Although a live-attenuated vaccine to prevent $\mathrm{HZ}$ was introduced in 2006 , it could be administered only to patients with RD prior to the start of immunosuppression or to those taking low-dose immunosuppressive therapies ${ }^{1,7,8}$. An adjuvanted, non-living recombinant zoster vaccine, Shingrix, was approved for use by Health Canada in 2017. Although limited data are available on the use of this vaccine in immunocompromised adults aged $\geq 50$ years, vaccination will likely provide some degree of protection from $\mathrm{HZ}$ reactivation ${ }^{10}$.

Data on the incidence of hepatitis B virus (HBV) infection in patients with RD are limited but do not suggest an increased incidence; however, the immunosuppressive features of treatment may put them at risk for HBV reactivation $^{11,12}$. Nevertheless, HBV vaccination guidelines in patients with RD follow those for the Canadian general population, with immunization recommended in patients with an increased risk of exposure ${ }^{1,12}$.

In the management of patients with RD, European, American, and Canadian guidelines recommend vaccination against several infectious agents, including influenza virus, pneumococcus, $\mathrm{HBV}$, and $\mathrm{HZ}^{1,7,8,12,13}$. Despite these recommendations, immunization coverage remains suboptimal. Influenza and pneumococcal vaccination rates vary widely by country, but seldom exceed $50 \%$ in patients with $\mathrm{RD}^{14-28}$. Additionally, low vaccination rates against $\mathrm{HZ}$ are reported in even older RD patients for whom vaccination would be recommended, independent of their underlying rheumatologic condition ${ }^{29,30}$. In cross-sectional survey-based studies, a lack of physician recommendation is cited as the principal reason for non-vaccination, with patient concerns of adverse effects and uncertainty about vaccine efficacy also contributing to low vaccine uptake ${ }^{15,17,19,24,25,26,28}$.

To improve the outcomes of patients with rheumatic diseases, we considered implementing a vaccination program in our academic center. In preparation for this, and in the absence of data on vaccination rates in rheumatology patients in Canada, we undertook this study to assess vaccination coverage and predictors of vaccination among a Canadian population of rheumatology patients in routine clinical care.

\section{MATERIALS AND METHODS}

Ethics. This study was approved by the Ethics Committee of the McGill University Health Center (MUHC; protocol number 14-386 GEN). Waiver of informed consent to publish this material was granted by the MUHC Research Ethics Board because this study fulfilled criteria for Quality Improvement Research.

Design. This cross-sectional study consisted of a patient-administered survey developed to assess vaccination coverage and patient-reported barriers for non-vaccination among rheumatology patients in routine clinical care (Supplementary Figure 1, available with the online version of this article). The survey was developed for our study by rheumatologists in English and forward-translated to French by a professional translator. A bilingual panel of rheumatologists resolved inadequate concepts of translation. There was no back-translation. Pretesting of the survey was done with 10 respondents who were systematically debriefed.

Study subjects. Consecutive adult patients presenting to a single-center tertiary care rheumatology clinic between May and September 2015 were asked to fill out a survey without identifiers. The patients self-identified as having RA (i.e., RA or juvenile idiopathic arthritis), systemic autoimmune rheumatic diseases (SARD; i.e., vasculitis, SLE, SSc, myositis), spondyloarthropathies [SpA; i.e., psoriatic arthritis (PsA), ankylosing spondylitis (AS)], or other diseases (OD; i.e., osteoarthritis, fibromyalgia, crystal arthropathies). The case definition for RA was based on self-reported diagnosis and use of DMARD or biologics, while for SARD and SpA it was solely based on self-reported diagnosis. Patients in the OD group were classified according to self-reported diagnosis and a lack of treatment with DMARD/biologics. Details of the diagnoses of each study group are presented in Supplementary Table 1 (available with the online version of this article).

Study variables. Data collected for this study were self-reported, including demographics (sex and age), diagnosis, disease duration, current treatment (corticosteroids, DMARD, biologics), comorbidities [cancer, diabetes, renal disease (not considered a comorbidity in patients with SLE)], and whether they were followed by a primary care physician (PCP; defined as a PCP seen at least once a year for routine care). In addition, information was collected regarding influenza vaccination in the previous year (2014-2015 influenza season) and vaccination status for pneumococcus, HBV, and HZ. We considered coverage for pneumococcal and $\mathrm{HBV}$ vaccination if patients indicated they had received at least 1 dose of a relevant vaccine. We did not collect information on the type of pneumococcal vaccine used. Canadian guidelines recommend influenza and pneumococcal vaccination in persons aged $\geq 65$ years and in those aged 18-64 years with at least 1 chronic medical condition, including patients with RD classified as having an immune disease/suppression ${ }^{31,32}$. Because patients aged 18-64 years in the OD group did not have a chronic medical condition, we reported influenza and pneumococcal vaccination rates only for those patients aged $\geq 65$ years in the OD group. Likewise, at the time of our study, Canadian guidelines recommended $\mathrm{HZ}$ vaccination in those aged $\geq 60$ years irrespective of risk; therefore, we reported $\mathrm{HZ}$ immunization rates in those patients aged $\geq 60$ years across the 4 disease groups ${ }^{8,12}$. Moreover, we collected information on whether patients were aware that they could be protected from the infectious agent through vaccination (yes/no), and whether a physician had ever recommended the vaccine (yes/no).

To establish baseline influenza and pneumococcal vaccination rates among the Quebec general population, we used data published by the National Public Health Institute of Quebec (INSPQ). The INSPQ reported Quebec general population influenza vaccination rates for the 2015-2016 season and pneumococcal vaccination rates for 2015 in those aged $\geq 65$ years ${ }^{28}$. No data were reported by the INSPQ regarding the Quebec influenza vaccination rates for the 2014-2015 season. There are no published data regarding $\mathrm{HBV}$ and $\mathrm{HZ}$ vaccination rates in the Quebec general population.

Personal non-commercial use only. The Journal of Rheumatology Copyright @ 2020 . All rights reserved. 
Statistical analysis. Descriptive statistics were used to summarize baseline characteristics of each disease group (RA, SARD, SpA, OD). Continuous variables were reported as mean $\pm \mathrm{SD}$, and categorical variables as frequency (\%) relative to the number for whom data were available.

Univariate logistical regression models were generated to evaluate the association between vaccination rates among all rheumatology patients in routine clinical care and explanatory variables, including sex, age, followed by a PCP, disease duration (yrs), comorbidities (cancer, diabetes, renal disease), treatment (DMARD, biologics, both, neither), awareness that they could be protected from the infectious agent through vaccination, vaccine recommendation by a physician, and disease group (RA, SARD, SpA, OD). Univariate predictors found to be significant at the $\mathrm{p}<0.20$ level were included in a multivariate model to determine potential independent predictors of vaccine uptake, while controlling for confounding. Sex, age, and disease group were chosen for inclusion a priori in multivariate analysis. $\mathrm{P}$ values $<0.05$ were considered statistically significant.

Analyses were performed using SPSS Statistics (IBM Corp.).

\section{RESULTS}

Study population. The study population included 352 adult patients presenting to a tertiary care rheumatology clinic between May and September 2015. Patients self-classified as RA: 136, SARD: 113, SpA: 47, OD: 56 (Supplementary Table 1, available with the online version of this article). The baseline characteristics of the patients are summarized in Table 1. The proportion of patients followed by a PCP was similar across the different groups (RA: $77.7 \%$, SARD: $80.4 \%$, OD: $82.7 \%$ ), and slightly lower for SpA $(65.2 \%)$. The presence of comorbidities (cancer, diabetes, renal disease) was greatest in the OD and RA groups (28.6\% and $25.7 \%$, respectively). The majority of RA and SARD patients was treated with DMARD (69.1\% and 61.1\%, respectively), while those with SpA were often taking neither DMARD nor biologics (48.9\%). Awareness that vaccination is protective against infection was highest for the influenza vaccination (RA: $88.8 \%$, SARD: $91.1 \%$, SpA: $91.5 \%$, OD: 100\%). Similarly, physician recommendation was greatest for the influenza vaccination (RA: 74.4\%, SARD: $77.3 \%$, SpA: 60.9\%, OD: $88.2 \%$ ). Among the RA, SARD, and SpA patients who received a recommendation for pneumococcal vaccination by a physician, rheumatologists more frequently made the recommendation than PCP (RA $59.2 \%$ vs $34.2 \%$, SARD $59.4 \%$ vs $28.1 \%$, SpA $85.7 \%$ vs $14.3 \%$ ).

Vaccination rates. Within the disease groups, vaccination rates are reported for patients who had an indication for vaccination (Table 2). Influenza and pneumococcal vaccination rates were greater in the Quebec general population compared to the RD groups. HBV vaccination was greatest in the SARD and SpA groups, while HZ immunization rates were below $30 \%$ for all groups.

Multivariate predictors of vaccination. Univariate predictors associated with influenza, pneumococcal, or HBV vaccination are shown in Supplementary Tables 2-4 (available with the online version of this article). For influenza vaccination, multivariate analysis demonstrated that older age (OR 1.03, $95 \%$ CI 1.01-1.05, $\mathrm{p}=0.01)$, physician recommendation $(\mathrm{OR}$ $8.56,95 \%$ CI $2.80-26.2, \mathrm{p}<0.001)$, and previous pneumo- coccal vaccination (OR 3.31, 95\% CI 1.71-6.40, p < 0.001) were predictors of vaccination (Table 3 ). Multivariate predictors of pneumococcal vaccination were physician recommendation (OR 314, 95\% CI 73.0-1353, p < 0.001) and previous influenza vaccination (OR 4.05, 95\% CI 1.36-12.0, $\mathrm{p}=0.01)$. Last, younger age (OR 0.96, 95\% CI 0.94-0.99, $\mathrm{p}=0.01$ ), awareness of the protection offered by HBV vaccination (OR 11.5, 95\% CI 2.49-52.8, p = 0.002), and HBV vaccination recommendation by a physician (OR 12.8, 95\% CI $5.27-31.1, \mathrm{p}<0.001$ ) were predictors of HBV vaccination in multivariate analysis (Table 3 ).

Owing to the limited numbers of patients vaccinated against HZ, it was not possible to perform univariate or multivariate analyses of predictors of $\mathrm{HZ}$ vaccination in this population.

\section{DISCUSSION}

Our study highlights the suboptimal vaccination coverage in ambulatory patients with RD. To our knowledge, this is the first such report in a Canadian setting, and it is consistent with published literature from the United States and Europe $^{14-27,29,30}$. As has been reported, we identified physician recommendation of vaccination as the strongest predictor of vaccination against influenza, pneumococcus, and HBV. This indicates that physicians play a central role in improving vaccination coverage in this at-risk population.

The Canadian National Immunization Strategy has set targets of $80 \%$ coverage for influenza and pneumococcal vaccination $^{33}$. Our data demonstrate suboptimal influenza and pneumococcal vaccination coverage in patients with rheumatic diseases, with vaccination rates below $50 \%$. Quebec general population influenza and pneumococcal vaccination rates are similarly below Public Health targets at $58.5 \%$ and $53.2 \%$, respectively ${ }^{28}$. Nevertheless, there is a gap in vaccine uptake between the Quebec general population and $\mathrm{RD}$ groups despite both having indications for vaccination.

Several factors may help explain the low vaccination rates for influenza and pneumococcal disease among the RD groups. As disease activity and patient monitoring take priority during clinical visits, rheumatologists may not convey the need for vaccination to their patients. PCP may be unaware of the need to vaccinate adult patients with rheumatic diseases at any age against influenza and pneumococcus, and may partially account for the gaps between the $\mathrm{RD}$ groups and the Quebec general population ${ }^{19}$. Moreover, PCP may be uncertain about the safety of vaccination in patients receiving immunosuppressive therapy. This was highlighted by our results, as a recommendation for pneumococcal vaccination was more often made by a rheumatologist than a PCP in the RD groups. In line with this observation, a similar study investigating factors affecting influenza and pneumococcal vaccination rates among patients with chronic inflammatory joint diseases (RA and SpA) in France found that rheumatologists were more likely to recommend vacci-

Personal non-commercial use only. The Journal of Rheumatology Copyright @ 2020 . All rights reserved. 
Table 1. Baseline characteristics of study subjects by disease group.*

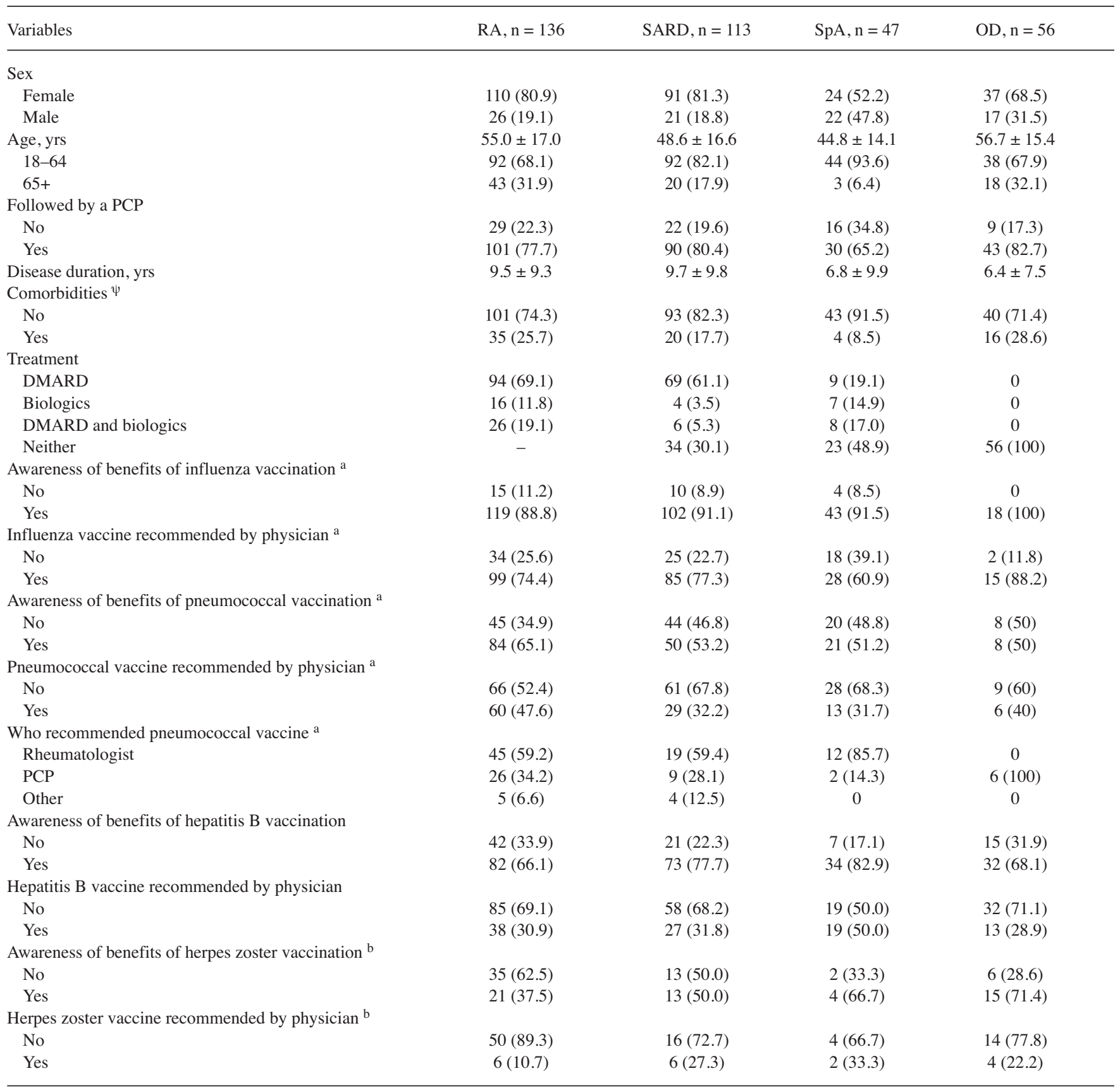

Values are frequency $(\%)$ or mean \pm SD. $*$ Where data were missing within categories, values were reported relative to the number for whom data were available.

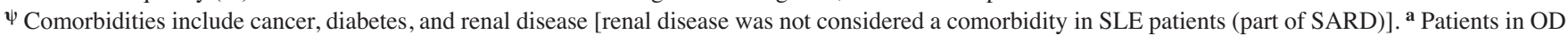
are $\geq 65$ years. ${ }^{\mathbf{b}}$ Patients in all groups are $\geq 60$ years. RA: rheumatoid arthritis; SARD: systemic autoimmune rheumatic diseases; SLE: systemic lupus erythematosus; SpA: spondyloarthropathies; OD: other diseases; DMARD: disease-modifying antirheumatic drugs; PCP: primary care physician.

nation compared to PCP (79\% vs $37 \%$ for influenza, and $78 \%$ vs $14 \%$ for pneumococcus $)^{24}$. A separate French study also demonstrated that in a cohort of patients with RA, SSc, AS, psoriasis, PsA, solid organ transplants, and malignant blood diseases, those followed by a specialist were more likely to be vaccinated against influenza than those followed by a
$\mathrm{PCP}^{34}$. In addition, we did not observe an association between being followed by a PCP and vaccine uptake in our multivariate analyses. Together, these findings point to a knowledge gap among PCP regarding vaccine recommendations in patients with RD. Nevertheless, in multivariate analyses examining associations between predictive factors

Personal non-commercial use only. The Journal of Rheumatology Copyright (C) 2020. All rights reserved. 
Table 2. Vaccination rates by disease group.

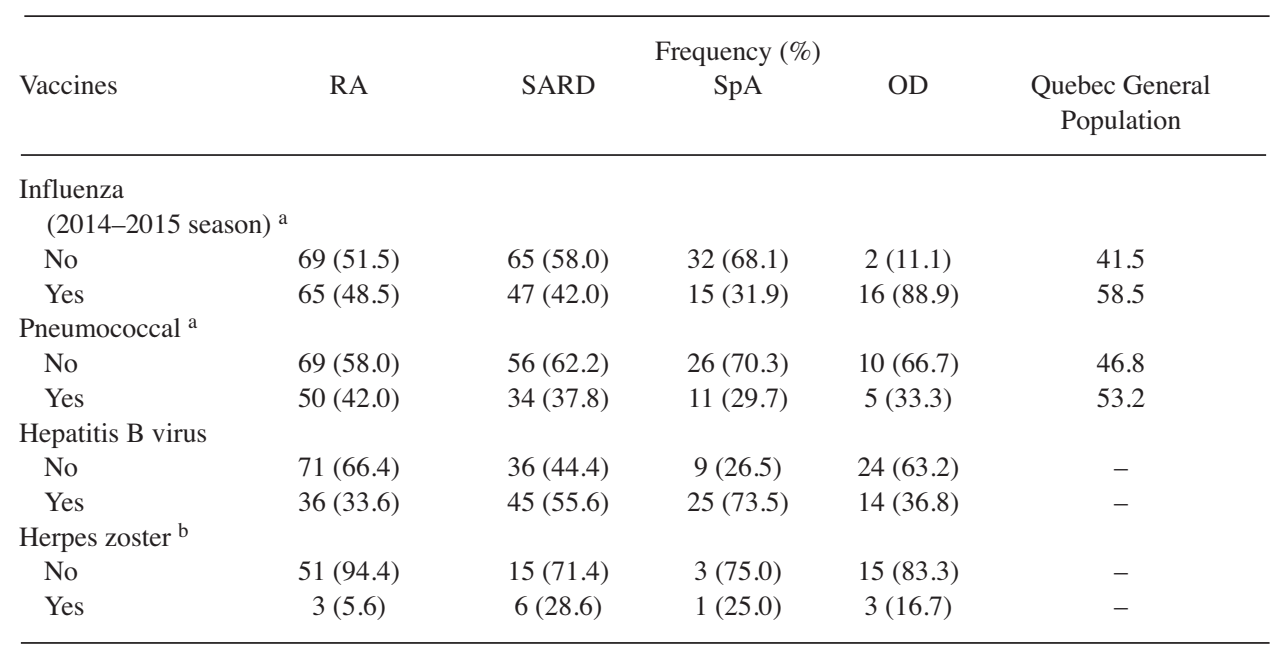

a Patients in the OD and Quebec general population groups are $\geq 65$ years. ${ }^{\mathbf{b}}$ Patients in all groups are $\geq 60$ years. RA: rheumatoid arthritis; SARD: systemic autoimmune rheumatic diseases; SpA: spondyloarthropathies; OD: other diseases.

and a physician recommendation for influenza or pneumococcal vaccination, we observed that having a PCP was associated with a recommendation for influenza but not pneumococcal vaccination (Supplementary Tables 5-6, available with the online version of this article). Thus, it may be that in our setting, PCP are more aware and comfortable with guidelines for influenza as compared to pneumococcal vaccination in patients with RD. Several factors could contribute to this, including the annual Public Health initiatives in support of influenza vaccination ${ }^{34}$.

Our data may also suggest a reluctance by PCP to recommend vaccination for rheumatology patients, possibly in deference to rheumatologists, whom PCP may perceive as better suited for the task. This was highlighted by a qualitative study aimed at identifying barriers and facilitators of vaccination among patients with $\mathrm{RA}^{35}$. PCP and nurse providers argued that rheumatologists should initiate the discussion about vaccination because their involvement in diagnosis and treatment may mean that their advice bears more weight ${ }^{35}$. This underscores the issue of perceived responsibility among providers regarding the discussion and administration of vaccines. Some physicians may choose to forgo a discussion on vaccination if they believe the onus lies with another provider. This attitude has been shown to lead to decreased vaccination coverage among other high-risk patients receiving care from both PCP and subspecialists $^{34,36,37,38}$. A lack of healthcare provider communication is central to this problem, and strategies to bridge this gap may prove successful in improving vaccine coverage.

In multivariate analysis, age was associated with decreased HBV vaccination (OR 0.96, 95\% CI 0.94-0.99, $\mathrm{p}=0.01$ ), echoing previously reported HBV vaccination data on patients with RD and at-risk individuals ${ }^{11,27}$. Consequently, HBV vaccination coverage was highest in patients grouped under SpA and SARD (73.5\% and 55.6\%, respectively), because they were also found to be younger than patients in the RA and OD groups (Table 1). The adoption of universal childhood vaccination programs in the mid-1990s in Canada, coupled with HBV testing as part of routine screening for sexually transmitted infections in sexually active persons, can account for much of our observations within these younger populations ${ }^{39}$. To further accentuate this point, the overall vaccination rate against $\mathrm{HBV}$ rises from $46.2 \%$ to $69.1 \%(\mathrm{n}=55)$ among all patients aged $\leq 35$ years (approximate cutoff age since the start of HBV childhood vaccination in Canada), while it falls to $39.7 \%(\mathrm{n}=204)$ in those aged $>35$ years (data not shown). Thus, while the future of HBV coverage is promising owing to routine childhood and infant vaccination programs, our results serve as a reminder not to neglect screening and vaccination in older patients at high risk of contracting $\mathrm{HBV}$, especially because we demonstrate a strong generational difference in vaccination rates.

Despite being at increased risk of infection from HZ, we observed poor vaccine coverage against $\mathrm{HZ}$ across all disease groups. Low levels of physician recommendation for $\mathrm{HZ}$ vaccination (overall proportion $=17.6 \%$, calculated from Table 1) most likely accounts for the low vaccination rates, especially because our multivariate analyses demonstrate the importance of physician recommendation in the context of vaccine uptake (Table 3). Patients who did not receive the $\mathrm{HZ}$ vaccine cited the lack of vaccine recommendation as the principal reason for non-vaccination, further supporting this finding (Supplementary Table 7, available with the online version of this article). Although this barrier has been removed with the introduction of a non-living $\mathrm{HZ}$ vaccine, there was likely a strong disinclination in 2015 from rheumatologists and PCP alike to recommend or administer a live

Personal non-commercial use only. The Journal of Rheumatology Copyright $\odot$ 2020. All rights reserved. 
Table 3. Multivariate analyses* to identify predictors of vaccination.

\begin{tabular}{|c|c|c|c|}
\hline Variables & OR & $95 \% \mathrm{CI}$ & $\mathrm{p}$ \\
\hline \multicolumn{4}{|c|}{ Influenza Vaccination $\delta$} \\
\hline Female & 0.71 & $0.32-1.60$ & 0.41 \\
\hline Age, yrs & 1.03 & $1.01-1.05$ & 0.011 \\
\hline Followed by a primary care physician & 1.69 & $0.76-3.75$ & 0.20 \\
\hline Comorbidities $\psi$ & 1.19 & $0.50-2.83$ & 0.69 \\
\hline Awareness of benefits of influenza vaccination & 4.28 & $0.75-24.4$ & 0.10 \\
\hline Influenza vaccine recommended by physician & 8.56 & $2.80-26.2$ & $<0.001$ \\
\hline Previous pneumococcal vaccination & 3.31 & $1.71-6.40$ & $<0.001$ \\
\hline Disease group (OD as ref. group) & & & 0.67 \\
\hline RA & 0.47 & $0.09-2.53$ & \\
\hline SARD & 0.38 & $0.07-2.12$ & \\
\hline SpA & 0.35 & $0.05-2.12$ & \\
\hline \multicolumn{4}{|c|}{ Pneumococcal Vaccination $\delta$} \\
\hline Female & 0.40 & $0.11-1.54$ & 0.18 \\
\hline Age, yrs & 1.01 & $0.97-1.04$ & 0.76 \\
\hline Treatment (neither as ref. group) & & & 0.78 \\
\hline DMARD & 0.89 & $0.16-4.95$ & \\
\hline Biologics & 2.52 & $0.25-25.9$ & \\
\hline DMARD and biologics & 0.89 & $0.13-6.23$ & \\
\hline Pneumococcal vaccine recommended by physician & 314 & $73.0-1353$ & $<0.001$ \\
\hline Previous influenza vaccination & 4.05 & $1.36-12.0$ & 0.01 \\
\hline Disease group (OD as ref. group) & & & 0.09 \\
\hline RA & 2.94 & $0.20-44.2$ & \\
\hline SARD & 14.5 & $0.94-225$ & \\
\hline SpA & 2.41 & $0.17-34.4$ & \\
\hline \multicolumn{4}{|c|}{ HBV Vaccination } \\
\hline Female & 0.51 & $0.20-1.34$ & 0.17 \\
\hline Age, yrs & 0.96 & $0.94-0.99$ & 0.01 \\
\hline Disease duration, yrs & 0.99 & $0.94-1.05$ & 0.75 \\
\hline Comorbidities $\psi$ & 1.30 & $0.44-3.83$ & 0.63 \\
\hline Awareness of benefits of HBV vaccination & 11.5 & $2.49-52.8$ & 0.002 \\
\hline HBV vaccine recommended by physician & 12.8 & $5.27-31.1$ & $<0.001$ \\
\hline Disease group (OD as ref. group) & & & 0.06 \\
\hline RA & 0.76 & $0.22-2.66$ & \\
\hline SARD & 2.74 & $0.74-10.1$ & \\
\hline SpA & 2.02 & $0.43-9.48$ & \\
\hline
\end{tabular}

* For multivariate analyses, sex, age, and disease group were included a priori. ${ }^{\delta}$ Patients in the RD groups are of any age; patients in OD group are $\geq 65 \mathrm{yrs}$. $\psi$ Comorbidities include cancer, diabetes, and renal disease [renal disease was not considered a comorbidity in SLE patients (part of SARD)]. RA: rheumatoid arthritis; SARD: systemic autoimmune rheumatic diseases; SLE: systemic lupus erythematosus; SpA: spondyloarthropathies; OD: other diseases; DMARD: disease-modifying antirheumatic drugs; HBV: hepatitis B virus; $\mathrm{RD}$ : rheumatic disease.

$H Z$ vaccination to immunosuppressed patients with RD. Some patients with RD could have safely received the living $\mathrm{HZ}$ vaccine, but a lack of safety and efficacy data regarding $\mathrm{HZ}$ vaccination in immunocompromised and $\mathrm{RD}$ patients was undoubtedly compounded by a lack of knowledge concerning $\mathrm{HZ}$ guideline recommendations and policies $^{2,8,29,30}$. An additional barrier to $\mathrm{HZ}$ vaccination with Zostavax II was patient cost, because the vaccine was not covered by the Quebec Immunization Program ${ }^{10}$. However, recent Health Canada approval of Shingrix, a non-living, recombinant subunit adjuvanted vaccine to prevent $\mathrm{HZ}$, may lead to improved $\mathrm{HZ}$ protection in patients with RD by overcoming structural, biological, and potential financial barriers to $\mathrm{HZ}$ vaccination ${ }^{10}$.
We identified several predictors of vaccination uptake in our multivariate analyses. Of note, physician recommendation of the vaccine was the strongest independent predictor of vaccination and the only factor consistently associated with vaccine uptake. This emphasizes the central role of the physician in the appropriate and effective vaccination of at-risk patients. It also adds to a growing body of literature placing physician recommendation as a crucial factor in patient vaccination ${ }^{15,19,24,26,28}$. Nevertheless, as outlined above, the notion of perceived responsibility for vaccination between PCP and subspecialists can lead to oversight and missed opportunities for vaccination. Improved collaboration and communication across healthcare professionals is imperative to bridging this gap in care.

Personal non-commercial use only. The Journal of Rheumatology Copyright @ 2020 . All rights reserved. 
Patient factors, including education and attitude, can affect vaccination rates. We show in our multivariate analyses that patient awareness of the protection offered by vaccination is associated with physician recommendation of influenza and pneumococcal vaccination (Supplementary Table 5-6, available with the online version of this article). Thus, patients who are more mindful about vaccination will better engage their physicians on the topic. Additionally, the principal reasons for non-vaccination against influenza cited by patients were disinterest and misconceptions regarding the vaccine (Supplementary Table 7). While we are conducting qualitative studies in RA to define the perceived barriers and facilitators of vaccine acceptance, interventions to improve vaccine acceptance among patients at high risk for vaccine-preventable diseases are greatly needed ${ }^{40}$.

Improving vaccination access is vital to enhancing vaccination uptake. The availability of specialized vaccine centers, immunization programs targeting at-risk populations, electronic medical record (EMR)-based alert systems, and staff education are strategies proven to increase influenza vaccination rates $30,41,42,43$. Where EMR-based solutions are not available, clinical reminders in paper charts and the use of standing order programs have also been shown to improve vaccine uptake $e^{44,45,46}$. Further, because of the strong association between physician recommendation and vaccine uptake, physician education is consistently a key component of vaccination improvement strategies ${ }^{30,47}$. Last, a willingness to evaluate vaccination practices within a clinic by routinely measuring and reporting vaccine coverage data is a crucial component in the improvement process ${ }^{20,21,22,48}$.

Our study has several limitations. First, there is the risk of recall bias because we relied on self-reported vaccination rates and diagnoses that were not verified by medical records. While this may have resulted in an overestimation of some vaccination rates and inappropriate categorization of patients, self-reporting has been shown to be a reliable method of vaccination and disease assessment ${ }^{49,50}$. In addition, by considering whether patients had ever been vaccinated against pneumococcus or HBV, we overestimated the number of appropriately immunized patients because both vaccines require more than 1 dose for optimal longterm protection ${ }^{19}$. Further work to address appropriate pneumococcal and HBV vaccination status in this vulnerable population would be of value. Moreover, our data reflect vaccination rates from a single academic center and may not be reflective of community rates. Because response rate data were not collected, nonresponder demographics are not available. Thus, despite survey availability to all patients presenting to the rheumatology clinic, certain patient populations were biased by our sample collection. This could include patients who are hesitant or reluctant to be vaccinated. If that was the case, the estimates we report may represent an overestimation of the real number of patients vaccinated. While comparator data from other Canadian centers are not available, our findings are in line with similar studies in Europe and the United States, suggesting that the quality gap in immunization coverage among rheumatology patients is not unique to our population ${ }^{14-27,29,30}$. Limited by our sample size, we used INSPQ data to establish baseline values for influenza and pneumococcal vaccination rates in the Quebec general population ${ }^{28}$. This proved useful because it allowed for the direct comparison of the vaccination rates against influenza and pneumococcal disease in our study to those of the Quebec general population with an indication for vaccination. Further, owing to small sample sizes in some of our groups, we observed some inflated OR and wide $95 \% \mathrm{CI}$ in our multivariate analyses (e.g., physician recommendation and pneumococcal vaccination; Table 3). Additional research with a larger sample size would be needed to provide more robust estimates of the association between vaccine recommendation by a physician and vaccine uptake. To the best of our knowledge, this is the first study examining vaccination rates in a population of Canadian rheumatology patients.

Despite national guidelines and recommendations for vaccination in rheumatology patients, we demonstrate suboptimal vaccination coverage against influenza, pneumococcus, HBV, and HZ. Physician recommendation of vaccination was the strongest independent predictor of vaccine uptake in our study, highlighting a central role for physician education, engagement, and collaboration toward the optimization of vaccination rates in this at-risk population.

\section{ACKNOWLEDGMENT}

We thank Maria C.B. Bardales, Stefanie Lutak, and Heather Wiseblatt for help with coordinating and recruiting patients.

\section{ONLINE SUPPLEMENT}

Supplementary material accompanies the online version of this article.

\section{REFERENCES}

1. van Assen S, Elkayam O, Agmon-Levin N, Cervera R, Doran MF, Dougados $\mathrm{M}$, et al. Vaccination in adult patients with auto-immune inflammatory rheumatic diseases: a systematic literature review for the European League Against Rheumatism evidence-based recommendations for vaccination in adult patients with autoimmune inflammatory rheumatic diseases. Autoimmun Rev 2011;10:341-52.

2. Rahier JF, Moutschen M, Van Gompel A, Van Ranst M, Louis E, Segaert $\mathrm{S}$, et al. Vaccinations in patients with immune-mediated inflammatory diseases. Rheumatology 2010;49:1815-27.

3. Westra J, Rondaan C, van Assen S, Bijl M. Vaccination of patients with autoimmune inflammatory rheumatic diseases. Nat Rev Rheumatol 2014;11:135-45.

4. Naz SM, Symmons DP. Mortality in established rheumatoid arthritis. Best Pract Res Clin Rheumatol 2007;21:871-83.

5. Blumentals WA, Arreglado A, Napalkov P, Toovey S. Rheumatoid arthritis and the incidence of influenza and influenza-related complications: a retrospective cohort study. BMC Musculoskelet Disord 2012;13:158.

6. Wotton CJ, Goldacre MJ. Risk of invasive pneumococcal disease in people admitted to hospital with selected immune-mediated diseases: record linkage cohort analyses. J Epidemiol Community Health 2012;66:1177-81. 
7. Harpaz R, Ortega-Sanchez IR, Seward JF; Advisory Committee on Immunization Practices (ACIP) Centers for Disease Control and Prevention (CDC). Prevention of herpes zoster: recommendations of the Advisory Committee on Immunization Practices (ACIP). MMWR Recomm Rep 2008;57:1-30.

8. Government of Canada. Update on the use of herpes zoster vaccine: an Advisory Committee Statement (ACS) National Advisory Committee on Immunization (NACI). [Internet. Accessed October 21, 2019.] Available from: www.canada.ca/en/ public-health/services/publications/healthy-living/ update-use-herpes-zoster-vaccine.html

9. Colombel JF. Herpes zoster in patients receiving JAK inhibitors for ulcerative colitis: mechanism, epidemiology, management, and prevention. Inflamm Bowel Dis 2018;24:2173-82.

10. Comité sur l'immunisation du Quebec. [Opinion on the appropriateness of adding the vaccination against shingles to the Quebec Immunization Program. Author: Committee on Immunization of Quebec (CIQ)]. [Article in French] [Internet. Accessed October 21, 2019.] Available from: catalogue.santecom.qc.ca/cgi-bin/koha/opac-detail.pl? biblionumber $=110411$

11. Feuchtenberger M, Schäfer A, Philipp Nigg A, Rupert Kraus M. Hepatitis B serology in patients with rheumatic diseases. Open Rheumatol J 2016;10:39-48.

12. Bombardier C, Hazlewood GS, Akhavan P, Schieir O, Dooley A, Haraoui B, et al; Canadian Rheumatology Association. Canadian Rheumatology Association recommendations for the pharmacological management of rheumatoid arthritis with traditional and biologic disease-modifying antirheumatic drugs: part II safety. J Rheumatol 2012;39:1583-602.

13. Singh JA, Saag KG, Bridges SL Jr, Akl EA, Bannuru RR, Sullivan MC, et al. 2015 American College of Rheumatology guideline for the treatment of rheumatoid arthritis. Arthritis Rheumatol 2016;68:1-26

14. Hmamouchi I, Winthrop K, Launay O, Dougados M. Low rate of influenza and pneumococcal vaccine coverage in rheumatoid arthritis: data from the international COMORA cohort. Vaccine 2015;33:1446-52.

15. Nguyen M, Lindegaard H, Hendricks O, Friis-Moller N. Factors associated with influenza and pneumococcal vaccine uptake among rheumatoid arthritis patients in Denmark invited to participate in a pneumococcal vaccine trial (Immunovax_RA). Scand J Rheumatol 2017;46:446-53.

16. Michel M, Vincent FB, Rio S, Leon N, Marcelli C. Influenza vaccination status in rheumatoid arthritis and spondyloarthritis patients receiving biologic DMARDs. Joint Bone Spine 2016;83:237-8.

17. Mouthon L, Mestre C, Bérezné A, Poiraudeau S, Marchand C, Guilpain $\mathrm{P}$, et al. Low influenza vaccination rate among patients with systemic sclerosis. Rheumatology 2010;49:600-6.

18. Drenkard C, Rask KJ, Easley KA, Bao G, Lim SS. Primary preventive services in patients with systemic lupus erythematosus: study from a population-based sample in Southeast U.S. Semin Arthritis Rheum 2013;43:209-16.

19. Lawson EF, Trupin L, Yelin EH, Yazdany J. Reasons for failure to receive pneumococcal and influenza vaccinations among immunosuppressed patients with systemic lupus erythematosus. Semin Arthritis Rheum 2015;44:666-71.

20. Pradeep J, Watts R, Clunie G. Audit on the uptake of influenza and pneumococcal vaccination in patients with rheumatoid arthritis. Ann Rheum Dis 2007;66:837-8.

21. Haroon M, Adeeb F, Eltahir A, Harney S. The uptake of influenza and pneumococcal vaccination among immunocompromised patients attending rheumatology outpatient clinics. Joint Bone Spine 2011;78:374-7.
22. Desai SP, Turchin A, Szent-Gyorgyi LE, Weinblatt M, Coblyn J, Solomon DH, et al. Routinely measuring and reporting pneumococcal vaccination among immunosuppressed rheumatology outpatients: the first step in improving quality. Rheumatology 2011;50:366-72.

23. Costello R, Winthrop KL, Pye SR, Brown B, Dixon WG. Influenza and pneumococcal vaccination uptake in patients with rheumatoid arthritis treated with immunosuppressive therapy in the UK: a retrospective cohort study using data from the Clinical Practice Research Datalink. PLoS One 2016;11:e0153848.

24. Brocq O, Acquacalda E, Berthier F, Albert C, Bolla G, Millasseau E, et al. Influenza and pneumococcal vaccine coverage in 584 patients taking biological therapy for chronic inflammatory joint: a retrospective study. Joint Bone Spine 2016;83:155-9.

25. Assala M, Groh M, Blanche P, Vinter C, Cohen P, Le Guern V, et al. Pneumococcal and influenza vaccination rates in patients treated with corticosteroids and/or immunosuppressive therapies for systemic autoimmune diseases: a cross-sectional study. Joint Bone Spine 2017;84:365-6.

26. Morel J, Czitrom SG, Mallick A, Sellam J, Sibilia J. Vaccinations in adults with chronic inflammatory joint disease: immunization schedule and recommendations for patients taking synthetic or biological disease-modifying antirheumatic drugs. Joint Bone Spine 2016;83:135-41.

27. Krasselt M, Ivanov JP, Baerwald C, Seifert O. Low vaccination rates among patients with rheumatoid arthritis in a German outpatient clinic. Rheumatol Int 2017;37:229-37.

28. Dubé É, Kiely M, Ouakki M. [Quebec survey on vaccination against seasonal flu and pneumococcus and the determinants of vaccination: 2016: investigation report.] [Article in French] [Internet. Accessed October 21, 2019.] Available from: catalogue.santecom.qc.ca/ cgi-bin/koha/opac-detail.pl?biblionumber $=96535$

29. Zhang J, Delzell E, Xie F, Baddley JW, Spettell C, McMahan RM, et al. The use, safety, and effectiveness of herpes zoster vaccination in individuals with inflammatory and autoimmune diseases: a longitudinal observational study. Arthritis Res Ther 2011;13:R174.

30. Sheth H, Moreland L, Peterson H, Aggarwal R. Improvement in herpes zoster vaccination in patients with rheumatoid arthritis: a quality improvement project. J Rheumatol 2017;44:11-7.

31. Government of Canada. Canadian immunization guide chapter on influenza and statement on seasonal influenza vaccine for 2017-2018. [Internet. Accessed October 21, 2019.] Available from: www.canada.ca/en/public-health/services/publications/ healthy-living/canadian-immunization-guide-statement-seasonalinfluenza-vaccine-2017-2018.html

32. Government of Canada. Page 16: Canadian immunization guide: part 4 - active vaccines. [Internet. Accessed October 21, 2019.] Available from: www.canada.ca/en/public-health/services/ publications/healthy-living/canadian-immunization-guide-part-4active-vaccines/page-16-pneumococcal-vaccine.html

33. Government of Canada. Vaccination coverage goals and vaccine preventable disease reduction targets by 2025 . [Internet. Accessed October 21, 2019.] Available from: www.canada.ca/en/ public-health/services/immunization-vaccine-priorities/ national-immunization-strategy/vaccination-coverage-goalsvaccine-preventable-diseases-reduction-targets-2025.html

34. Loubet $\mathrm{P}$, Kernéis $\mathrm{S}$, Groh $\mathrm{M}$, Loulergue $\mathrm{P}$, Blanche $\mathrm{P}$, Verger $\mathrm{P}$, et al. Attitude, knowledge and factors associated with influenza and pneumococcal vaccine uptake in a large cohort of patients with secondary immune deficiency. Vaccine 2015;33:3703-8.

35. Boucher VG, Pelaez S, Lavoie KL, Colmegna I. Perceived barriers and facilitators of vaccination acceptance among rheumatoid arthritis patients from the perspective of physician and nurse providers [abstract]. J Rheumatol 2018; 45:964-1069.

36. Rickert D, Santoli J, Shefer A, Myrick A, Yusuf H. Influenza

Personal non-commercial use only. The Journal of Rheumatology Copyright @ 2020 . All rights reserved. 
vaccination of high-risk children: what the providers say. Am J Prev Med 2006;30:111-8.

37. Davis MM, McMahon SR, Santoli JM, Schwartz B, Clark SJ. A national survey of physician practices regarding influenza vaccine. J Gen Intern Med 2002;17:670-6.

38. Jessop AB, Dumas H, Moser CA. Delivering influenza vaccine to high-risk adults: subspecialty physician practices. Am J Med Qual 2012;28:232-7.

39. Public Health Agency of C. Epi-update: brief report: Hepatitis B infection in Canada. [Internet. Accessed October 21, 2019.] Available from: www.phac-aspc.gc.ca/id-mi/pdf/hepB-eng.pdf

40. Gosselin Boucher V, Colmegna I, Gemme C, Labbe S, Pelaez S, Lavoie KL. Interventions to improve vaccine acceptance among rheumatoid arthritis patients: a systematic review. Clinical Rheumatol 2019; 38:1537-44.

41. Ledwich LJ, Harrington TM, Ayoub WT, Sartorius JA, Newman ED. Improved influenza and pneumococcal vaccination in rheumatology patients taking immunosuppressants using an electronic health record best practice alert. Arthritis Rheum 2009;61:1505-10.

42. Top KA, Zafack J, De Serres G, Halperin SA. Canadian paediatricians' approaches to managing patients with adverse events following immunization: the role of the Special Immunization Clinic network. Paediatr Child Health 2014;19:310-4.

43. Thomas RE, Lorenzetti DL. Interventions to increase influenza vaccination rates of those 60 years and older in the community. Cochrane Database Syst Rev 2018;5:CD005188.
44. Desai SP, Lu B, Szent-Gyorgyi LE, Bogdanova AA, Turchin A, Weinblatt $\mathrm{M}$, et al. Increasing pneumococcal vaccination for immunosuppressed patients: a cluster quality improvement trial. Arthritis Rheum 2013;65:39-47.

45. McKibben LJ, Stange PV, Sneller VP, Strikas RA, Rodewald LE; Advisory Committee on Immunization Practices. Use of standing orders programs to increase adult vaccination rates: recommendations of the Advisory Committee on Immunization Practices. MMWR Recomm Rep 2000;49:15-6.

46. Warner EA, Seleznick MJ. Using medical record reminders to improve pneumococcal vaccination rates. Joint Jt Comm J Qual Saf 2004;30:331-4.

47. Bero LA, Grilli R, Grimshaw JM, Harvey E, Oxman AD, Thomson MA. Closing the gap between research and practice: an overview of systematic reviews of interventions to promote the implementation of research findings. The Cochrane Effective Practice and Organization of Care Review Group. BMJ 1998;317:465-8.

48. Subesinghe S, Rutherford AI, Ibrahim F, Harris H, Galloway J. A large two-centre study in to rates of influenza and pneumococcal vaccination and infection burden in rheumatoid arthritis in the UK. BMC Musculoskelet Disord 2016;17:322.

49. Irving SA, Donahue JG, Shay DK, Ellis-Coyle TL, Belongia EA. Evaluation of self-reported and registry-based influenza vaccination status in a Wisconsin cohort. Vaccine 2009;27:6546-9.

50. Rasooly I, Papageorgiou AC, Badley EM. Comparison of clinical and self reported diagnosis for rheumatology outpatients. Ann Rheum Dis 1995;54:850-2. 